Supplement of

\title{
Lehrentwurf: Die MOSAiC-Expedition und das Gradnetz der Erde - Wie können Lehrkräfte als Expeditionsteilnehmer die Polarforschung in die Klassenräume bringen?
}

Rainer Lehmann and Friederike Krüger

Correspondence to: Rainer Lehmann (rainer.lehmann@uni-flensburg.de)

The copyright of individual parts of the supplement might differ from the article licence. 
Für diese Aufgabe musst du wissen, wie man Koordinaten liest!

\section{Längen- und Breitengrade}

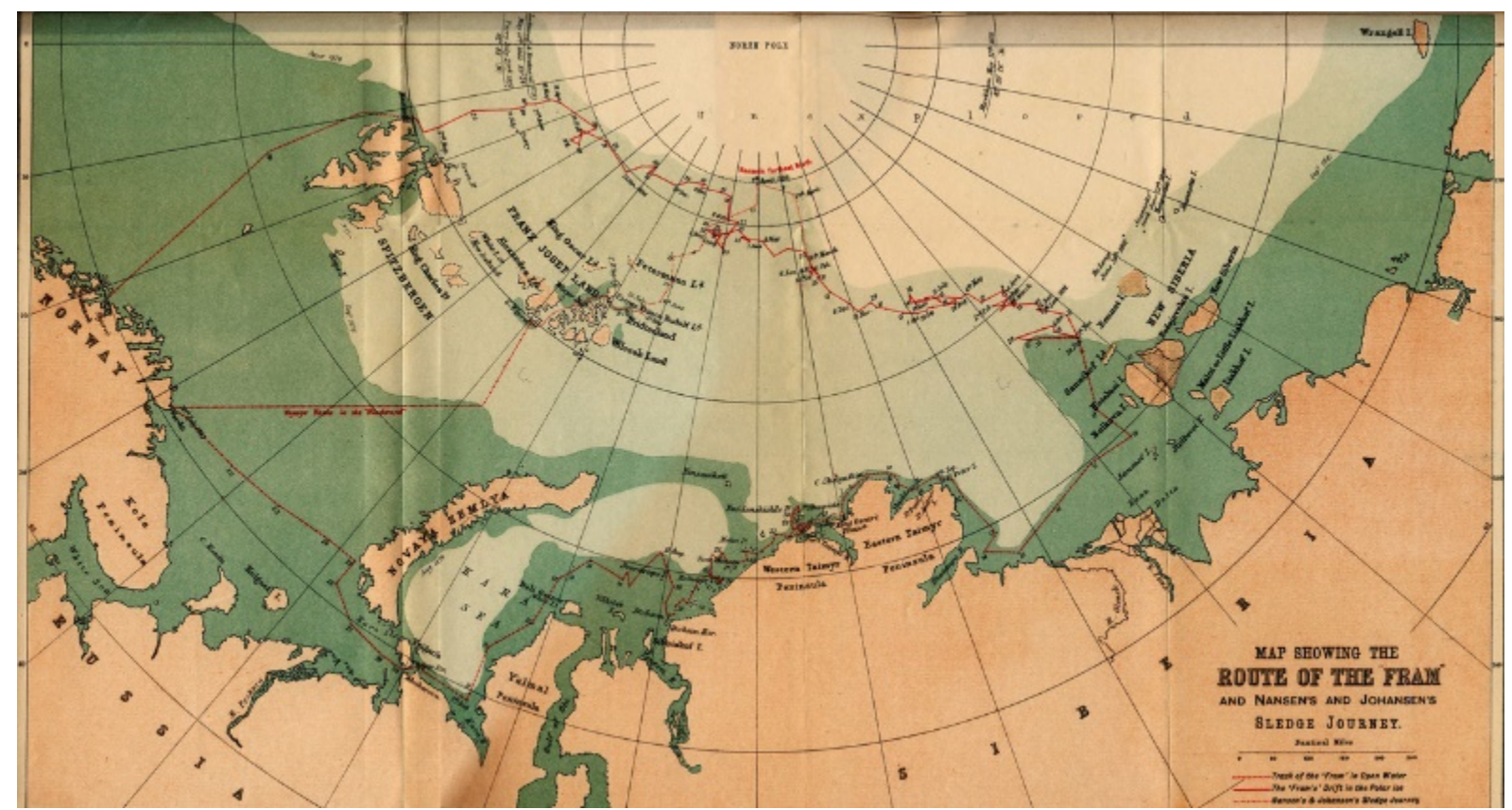

Abb. 1: Historische Karte der Fram-Expedition. Bild: Norwegian Polar Institute/Fram Centre (s.a. Fridtjof Nansen (1896): In Nacht und Eis) https://www.helmholtz.delerde_und_umwelt/fridtjof-nansen-auf-dem-weg-zum-nordpol/

Zwischen 1893 und 1896 unternahm Fridtjof Nansen die abenteuerliche Reise in die Arktis seiner Annahme folgend, dass es eine Polardrift gibt, die sein im Eis eingefrorenes Schiff einige Kilometer pro Tag über den Pol hinweg transportieren würde. Auf der Karte könnt ihr seinen Weg nachvollziehen (Abb. 1). Am 20.09.2019 machte sich das deutsche Polarforschungsschiff POLARSTERN auf den Weg, um dem Vorbild Nansen zu folgen und mithilfe der Transpolardrift innerhalb eines Jahres den Nordpolbereich zu überqueren und um die Klimaprozesse der Arktis besser zu verstehen. Begleitet wurde die POLARSTERN zu Beginn der Expedition im September und Oktober 2019 von dem russischen Schiff Akademik Fedorov. Auf ihm waren weitere Expeditionsteilnehmer*innen untergebracht sowie Nachschub an Lebensmitteln und Treibstoff.

In der untenstehenden Tabelle findest du die Koordinaten der ersten Fahrttage. Trage die Koordinaten der Akademik Fedorov in die Karte ein!

\begin{tabular}{|lll|}
\hline Tag & Breitengrad & Längengrad \\
& & \\
22.09. & $\mathrm{~N} 73^{\circ} 56^{\prime}$ & $\mathrm{O} 31^{\circ} 11^{\prime}$ \\
23.09. & $\mathrm{~N} 77^{\circ} 35^{\prime}$ & $\mathrm{O} 45^{\circ} 35^{\prime}$ \\
24.09. & $\mathrm{~N} 78^{\circ} 58^{\prime}$ & $\mathrm{O} 63^{\circ} 59^{\prime}$ \\
25.09. & $\mathrm{~N} 81^{\circ} 35^{\prime}$ & $\mathrm{O} 86^{\circ} 58^{\prime}$ \\
26.09. & $\mathrm{~N} 81^{\circ} 40^{\prime}$ & $\mathrm{O} 110^{\circ} 43^{\prime}$ \\
27.09. & $\mathrm{~N} 82^{\circ} 22^{\prime}$ & $\mathrm{O} 120^{\circ} 28^{\prime}$ \\
28.09. & $\mathrm{~N} 84^{\circ} 42^{\prime}$ & $\mathrm{O} 128^{\circ} 35^{\prime}$ \\
29.09. & $\mathrm{~N} 85^{\circ} 48^{\prime}$ & $\mathrm{O} 124^{\circ} 13^{\prime}$ \\
30.09. & $\mathrm{~N} 85^{\circ} 46^{\prime}$ & $\mathrm{O} 123^{\circ} 20^{\prime}$ \\
01.10. & $\mathrm{~N} 85^{\circ} 44^{\prime}$ & $\mathrm{O} 124^{\circ} 55^{\prime}$ \\
02.10. & $\mathrm{~N} 85^{\circ} 10^{\prime}$ & $\mathrm{O} 134^{\circ} 21^{\prime}$ \\
\hline
\end{tabular}

Tipp: Ein Breitengrad wird in 60 Minuten unterteilt, um die Position noch genauer anzugeben. Und jede Minute wieder in 60 Sekunden. Aber die wurden im Beispiel weggelassen. Man spricht also am 22.09. von 73 Grad und 56 Minuten!

Unterrichtsmaterial erstellt von Friederike Krüger, entstanden im Rahmen der MOSAiC-Expedition und der MOSAiC School durch Unterstützung des Alfred-Wegener-Instituts. Das Material darf unter Nennung der Ursprungsquelle frei verwendet werden. Weiteres Material unter mosaic-expedition.org. 
Erklärung: Koordinaten geben wie beim „Schiffe Versenken-Spiel“ die exakte Lage eines Punktes auf dem Gradnetz der Erde an. Das Gradnetz der Erde kannst du dir vorstellen wie ein Orangennetz, in dem die Erde liegt. Das Gradnetz besteht aus parallel zum Äquator verlaufenden Breitengraden (der Äquator hat $0^{\circ}$ und dann zählt man 90 Breitengrade bis zum Nordpol und genau 90 Breitengrade bis zum Südpol. Insgesamt gibt es also 180 Breitengrade. Je nachdem, ob der gesuchte Punkt auf der Nordhalbkugel oder auf der Südhalbkugel liegt, wird die Angabe mit N (Nördliche Breite) oder S (Südliche Breite) betitelt. Damit man die Lage noch genauer beschreiben kann, gibt es senkrecht verlaufende Längengrade, die in Greenwich (Londoner Stadtteil) jeweils beginnen und an den Polen zusammentreffen. Es verlaufen 180 Längengrade in östlicher Richtung (östliche Länge) und 180 Längengrade in westlicher Richtung (westliche Länge). Insgesamt gibt es also 360 Längengrade und 180 Breitengrade, die sich kreuzen und so Schnittpunkte angeben, über die man Positionen angeben und finden kann. Jeder Punkt auf der Erde lässt sich verorten. In der Schifffahrt ist das besonders wichtig, weil man nicht einfach sagen kann „wir sind 12 Kilometer von einem bestimmten Ort entfernt."

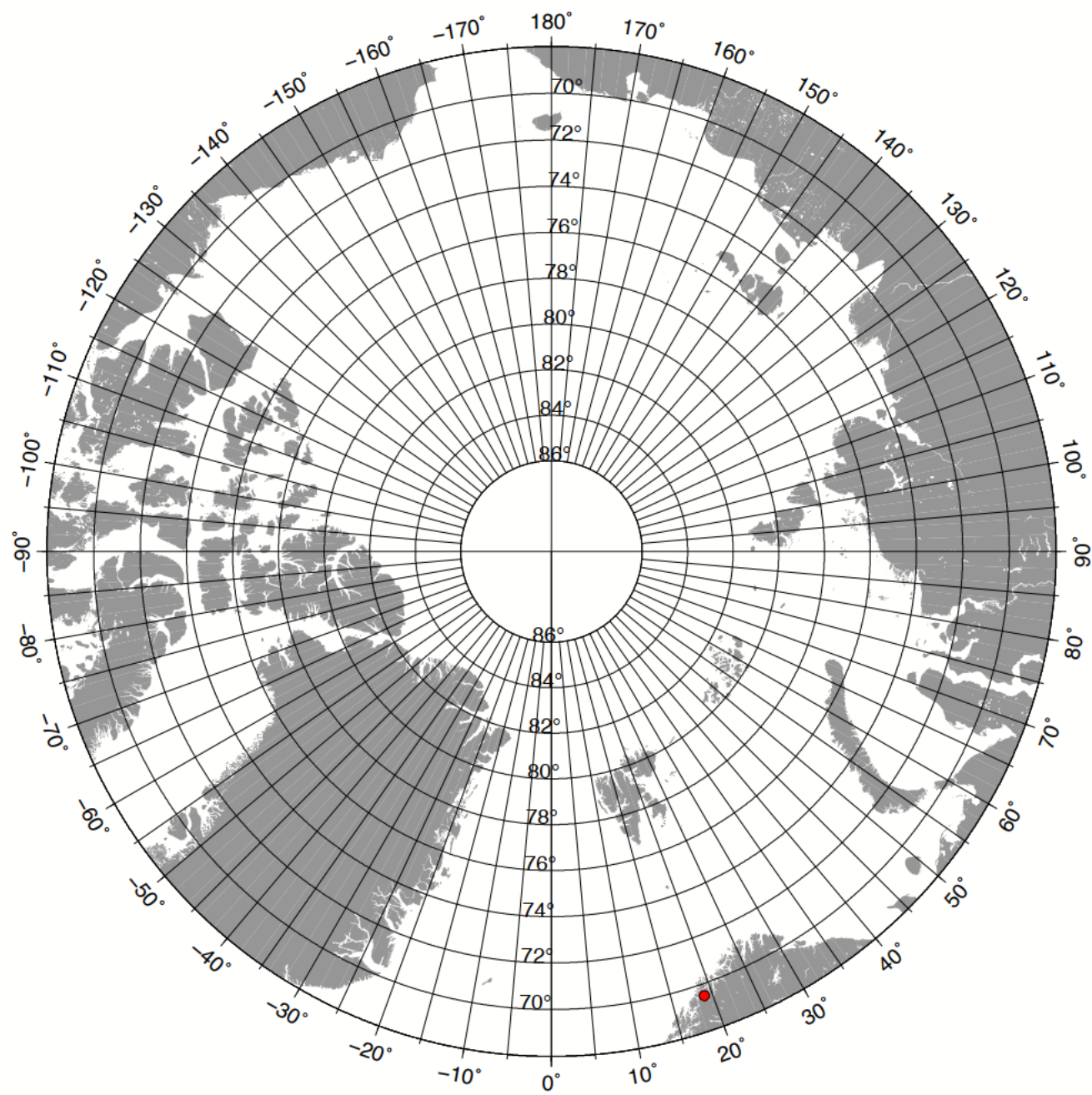

Abb. 2: Karte des Nordpolargebietes mit Längen- und Breitengraden (Vera Schlindwein, AWI)

Unterrichtsmaterial erstellt von Friederike Krüger, entstanden im Rahmen der MOSAiC-Expedition und der MOSAiC School durch Unterstützung des Alfred-Wegener-Instituts. Das Material darf unter Nennung der Ursprungsquelle frei verwendet werden. Weiteres Material unter mosaic-expedition.org. 


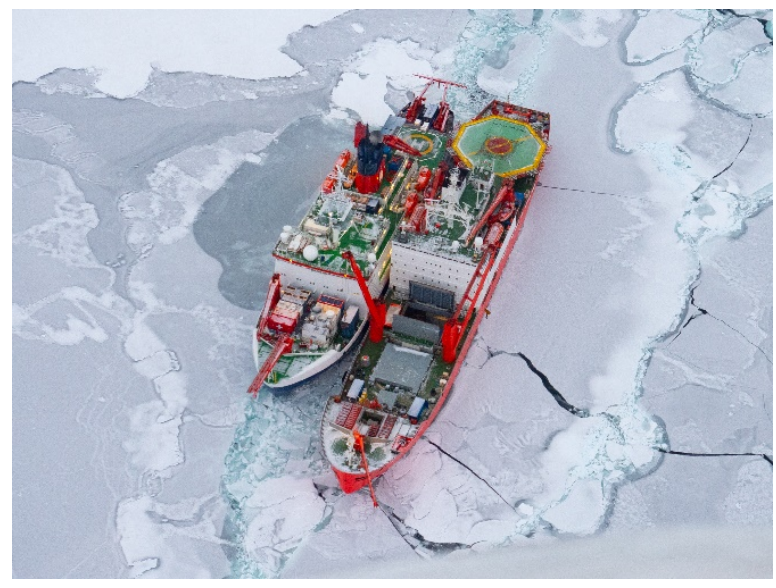

Abb. 3: Foto der Polarstern und Akademik Fedorov bei ihrem Treffen am 02. Oktober 2019 (Jan Rohde)

\section{Zusatzaufgabe:}

Mitten in der Arktis trafen sich die beiden Schiffe zum Austausch von 600 Tonnen Schiffsdiesel, Lebensmitteln und Expeditionsteilnehmer*innen am 2. Oktober. Denn zuvor hatte man die richtige Scholle gefunden und konnte nun die Expedition beginnen. Zeichne nun mithilfe der Koordinaten der POLARSTERN vom 1. und 2. Oktober die Routen beider Schiffe zum Treffpunkt ein!

\begin{tabular}{|c|c|c|}
\hline atum & Breitengrad & \\
\hline 01.10. & $\mathrm{~N} 85^{\circ} 20^{\prime}$ & 01 \\
\hline 2.10 & N 8510' & O $134^{\circ} 22$ \\
\hline
\end{tabular}

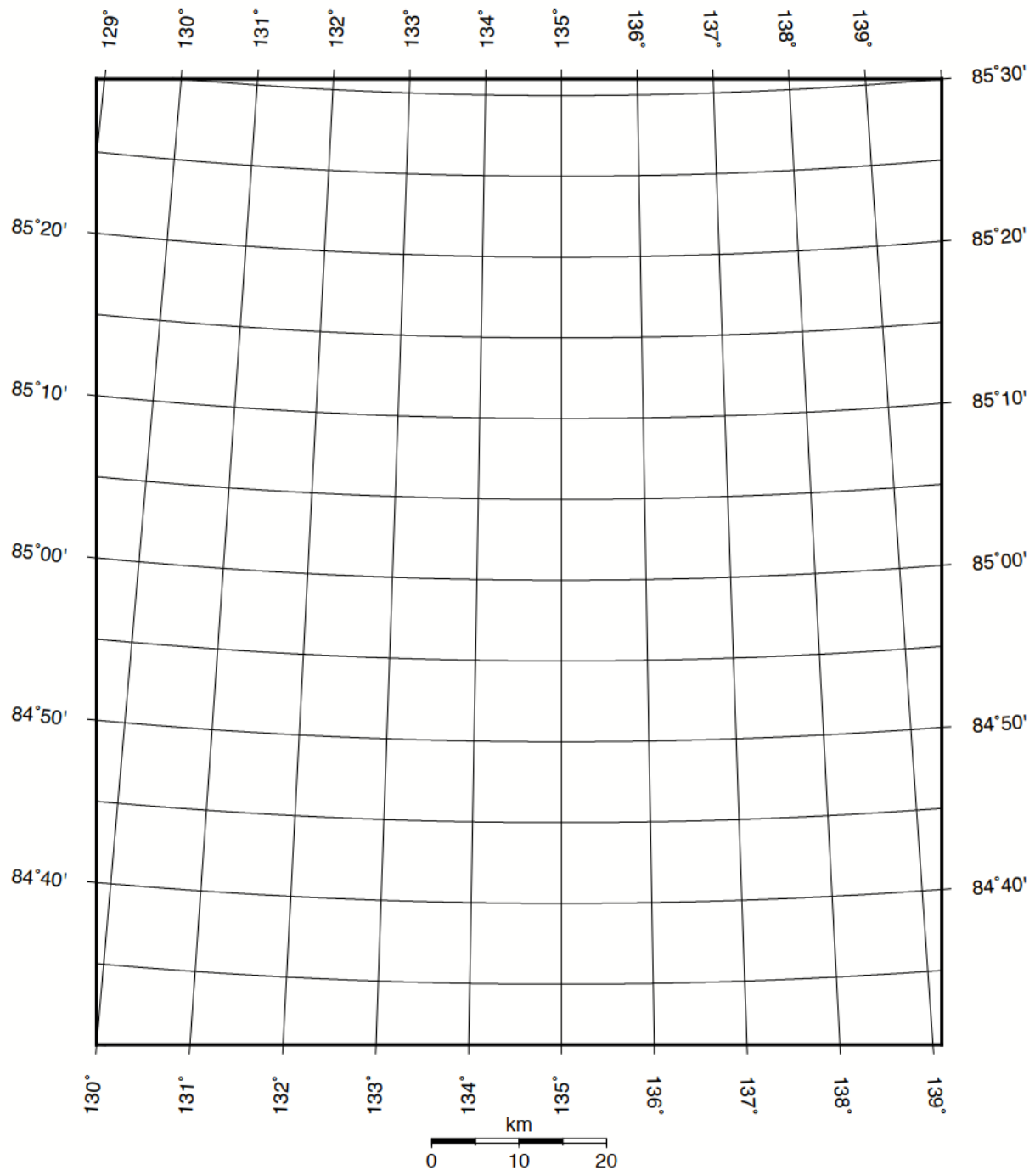

Abb. 4: Kartenausschnitt des Nordpolargebietes mit Längen- und Breitengraden (Vera Schlindwein, AWI)

Unterrichtsmaterial erstellt von Friederike Krüger, entstanden im Rahmen der MOSAiC-Expedition und der MOSAiC School durch Unterstützung des Alfred-Wegener-Instituts. Das Material darf unter Nennung der Ursprungsquelle frei verwendet werden. Weiteres Material unter mosaic-expedition.org. 